\title{
PCG-Delineator: an Efficient Algorithm for Automatic Heart Sounds Detection in Fetal Phonocardiography
}

\author{
Annachiara Strazza, Agnese Sbrollini, Valeria di Battista, Rita Ricci, Letizia Trillini, \\ Ilaria Marcantoni, Micaela Morettini, Sandro Fioretti, Laura Burattini \\ Università Politecnica delle Marche \\ Ancona, Italy
}

\begin{abstract}
Fetal phonocardiography (FPCG) is a non-invasive electronic recording of the acoustic cardiac signals. Unfortunately, FPCG is hidden by high-amplitude noise which makes detection of FPCG waveforms challenging. Aim of the study is to propose PCG-Delineator as an algorithm for automatic detection of the first and second heart sound ( $S 1$ and $S 2$, respectively) from FPCG. To this aim, 37 simulated FPCG tracings (Physionet) are filtered by a wavelet-based procedure (4 ${ }^{\text {th }}$ order Coiflets mother wavelet with 7 decomposition levels) to erase noise. Successively, S1 and S2 are detected. S1 detection procedure is threshold-based (threshold $=30 \%$ of the filtered FPCG signal maximum amplitude), under the condition that 40ms separate two consecutive S1 sounds. S2 detection procedure is also threshold-based, but under the conditions that $S 2$ has to fall 100ms after preceding S1 and 200ms before successive S1, and that S2 has to have an amplitude lower than $80 \%$ that of preceding S1. Sensitivity ( $S E)$ and positive predictive values $(P P V)$ were computed. Results indicate that PCG-Delineator was able to reduce noise (our SNR: from $-1.1 \div 7.4 \mathrm{~dB}$ to $\left.12.9 \div 17.9 \mathrm{~dB} ; P<10^{-14}\right)$ and to accurately detect both $S 1$ (SE: 88\%; PPV: 91\%) and S2 (SE: 77\%; PPV:99\%). In conclusion, PCG-Delineator is an efficient algorithm for automatic heart sounds detection in FPCG.
\end{abstract}

\section{Introduction}

Fetal phonocardiography (FPCG) is a low-cost and non-invasive technique for the detection of fetal heart sounds (fHSs), recording the vibroacoustic signals from the maternal abdomen. FPCG signals analysis provides precious diagnostic information for proper fetal wellbeing assessment during the period of pregnancy [1].

Under normal condition, fHSs can be characterized by two major audible sounds for each cardiac cycle: the first heart sound (S1) and the second heart sound (S2) [2]. The S1 sound, marking ventricular systole onset, is due to the sudden closure of atrioventricular valves. This sound is characterized of two internal components: the mitral component, associated with the closure of the mitral valve, and the tricuspid component, associated with the closing of the tricuspid valve. The S2 sound, marking diastole onset, is made up of two components: the aortic component, corresponding to the closure of the aortic valve, and the pulmonary component, corresponding of the closure of the pulmonary valve. S1 sound typically contains low frequency with longest duration and highest intensity. Indeed, S2 sound presents smoother morphology with high frequency and shorter duration than the S1, making harder the detection of its location [3]. Unfortunately, fHSs are very weak acoustic signals and they are heavily loaded by external acoustic signals and electrical noise interference, due to maternal heart sounds, digestive sounds, breathing, maternal and fetus respiration movements. [4,5]. Thus, determination of fHSs raises serious signal processing issues, due also to non-stationary nature of FPCG signals.

The development of an accurate and robust technique to detect fHSs has been since long researched. Many methods have been developed for FPCG segmentation and fHSs detection. Amplitude and/or time thresholdbased methods [6,7] probabilistic models-based methods $[8,9]$, and energy-based methods $[10,11]$ are developed to detect fHSs, but not considering the noisy environment of the FPCG signals. Thus, in a noisy environment, fHSs detection remains a challenging task and it is difficult to detect them accurately. Indeed, it is necessary to erase noise from the signals in order to make FPCG clinically usable. In this work, wavelet transformation (WT), a well-known denoising technique, have been proposed to filter FPCG [12,13]. In time-frequency analysis, WT processing is a useful tool for extracting local timefrequency information from a nonstationary signal, such as FPCG. Thus, aim of the present study is to propose PCG-Delineator as a novel and efficient algorithm to achieve a clear separation and detection of the fHSs from WT denoised FPCG signal.

\section{Materials and methods}

\subsection{Data}

Data consisted of 37 FPCG simulated tracings corrupted by different levels of noise, typically found in real recordings. Signals were generated as sequences of 
simulated heart sounds, corrupted by additive white Gaussian noise. All recordings belong to the "Simulated Fetal PCGs database" [14] of PhysioNet [15] freely accessible on the web under the ODC Public Domain Dedication and License v1.0. The database may be used without further ethical committee approval. Simulated PCGs are 8 min long (sampling frequency: $1 \mathrm{kHz}$ ), characterized by mean fHR of $140 \mathrm{bpm}$ and SNR included between $-26.7 \mathrm{~dB}$ and $-4.4 \mathrm{~dB}$.

\subsection{PCG-Delineator}

PCG-Delineator is an efficient algorithm for automatic HSs detection in FPCG. PCG-Delineator was implemented in Matlab; its block diagram is depicted in Figure 1. It operates in two consecutive steps, noise removal by WT filtering, and fHSs iterative detection. A detailed description of each step is reported below.

Wavelet Transformation Filtering. Typically, FPCG signals are heavily contaminated by noise from various sources, due to fetus movement and mother heart sound, breathing, and muscular movements. In order to make FPCG clinically suitable to detect cardiac sounds, it is necessary to remove noise from the signals. Thus, PCGDelineator performed a FPCG filtering by means of a WT-based procedure, a flexible approach to signal decomposition (WT denoising, Figure 1). WT is a timefrequency processing method that quantifies temporal changes of the frequency content of non-stationary signals [16]. WT of the input signal $x(t)$ is defined as (Eq.1):

$$
\mathrm{CWT}_{\mathrm{x}(\mathrm{a}, \mathrm{b})}=\int \mathrm{x}(\mathrm{t}) \Psi_{\mathrm{a}, \mathrm{b}}^{*}(\mathrm{t}) \mathrm{dt} \quad \mathrm{a} \neq 0
$$

where the basis function $\Psi_{\mathrm{a}, \mathrm{b}}(\mathrm{t})$ is the mother wavelet, featured by scale and time-shift parameters, $a$ and $b$, respectively (Eq.2):

$$
\psi_{a, b}(t)=\frac{1}{\sqrt{a}} \psi\left(\frac{t-b}{a}\right) .
$$

$\Psi_{a, b}(t)$ is the transforming function (mother wavelet) that can be used for signal decomposition. WT approach offers better temporal resolution and scale (frequency) resolution when analyzing high frequency component and low frequency components respectively. WT decomposes a signal into several multiresolution coefficients and performs a series of high- and low-pass filter operations followed by down-sampling. WT preserves signal by operating only on those selected regions of the bandwidth that need filtering. Denoising algorithm uses statistical regression of noisy coefficients over time to obtain a nonparametric estimation of the reconstructed signal without noise. Thus, PCG-Delineator for denoising decomposed the corrupted signal into several levels. The decomposition processing allows to remove the level which seriously corrupted by noise. In level decomposition, wavelet coefficients tend to be much larger than those due to noise. Thus, coefficients below a certain level are regarded as noise and they are thresholded out. Then, the signal can be reconstructed without significant loss of information in the signal content. As WT is sensitive to noise level, WT-procedure requires appropriate selection of wavelet family, level of decomposition, and threshold for having better accuracy. In this work, decomposition, denoising and reconstruction were performed by mother wavelet Coiflets of $4^{\text {th }}$ order with 7 levels of decomposition and soft threshold, providing the higher result for signal to noise ratio for all types of FPCG signal. Details of wavelet denoising algorithm are reported in [13].

fHSs Iterative Detection. According to PCGDelineator, S1 and S2 sounds are detected from WTdenoised FPCG. PCG-Delineator allowed to recognize all heart spikes and to identify which of them are S1 and which are S2. The PCG-Delineator algorithm to detect S1 and $\mathrm{S} 2$ sounds is a threshold-based. Initially, $\mathrm{S} 1$ detection procedure set an amplitude threshold as $30 \%$ of the filtered FPCG signal maximum amplitude, under the condition that at least $40 \mathrm{~ms}$ separate two consecutive S1 sounds. This time condition allows to remove possible $\mathrm{S} 1$ detections with no physiological meaning. In literature, it has been reported that, in non-pathological cases, S2 follow S1 in a heart cycle [4]. Consequently, S2 identification procedure, which is also based on a timethreshold, is performed after S1 detection (Figure 1). Specifically, S2 identification is based on the consideration that diastolic duration (i.e. time distance between S2 and S1 sounds) is longer than systolic duration (i.e. time distance between $\mathrm{S} 1$ and $\mathrm{S} 2$ sounds) [17]. Thus, S2 detection procedure is threshold-based in both time and amplitude, under the conditions that $\mathrm{S} 2$ has to fall at least $100 \mathrm{~ms}$ after preceding $\mathrm{S} 1$ and at most $200 \mathrm{~ms}$ before successive $\mathrm{S} 1$, and that $\mathrm{S} 2$ has to have an amplitude lower than $80 \%$ that of preceding S1 (S2 detection, Figure 1).

PCG-Delineator works iteratively: first, all potential $\mathrm{S} 1$ peaks are identified; then, distances between $\mathrm{S} 1$ peaks are computed to make a further control in time, in order to find all the S1 peaks that were not included in the first evaluation; once all S1 peaks have been localized and confirmed, S2 peaks are identified; eventually, S2 are confirmed by verifying their amplitude.

\subsection{Statistics}

Since original simulated signals and noise amplitudes were not available to reproduce the signal-to-noise ratio (SNR) values reported in the database, they were recomputed according to the definition reported in [13]. In order to evaluate their correctness, the Pearson 
correlation coefficient ( $\rho$ ) was computed. Automatic S1 and S2 detections were compared against provided

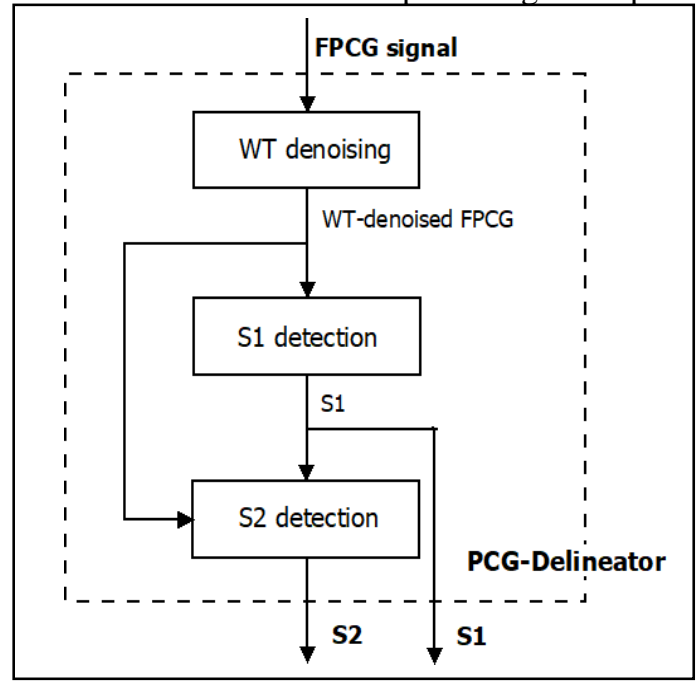

Figure 1. The block diagram of PCG-Delineator.

manual annotations and then they were classified as true positives, false positives and false negatives in order to quantify detection accuracy by means of sensitivity (SE) and positive predictive value (PPV).

\section{Results}

Computed SNR values ranged from $-1.1 \mathrm{~dB}$ to $7.4 \mathrm{~dB}$ and were strongly correlated $\left(\rho=1.0 ; P<10^{-200}\right)$ with those provided in the database. Thus, they were reliable to measure signal quality improvements after WT noise filtration by PCG-Delineator.

Results indicate that PCG-Delineator was able to drastically reduce noise. Indeed, after its application, SNR increased significantly $\left(12.9 \div 17.9 \mathrm{~dB} ; \mathrm{P}<10^{-14}\right)$. A qualitative example of raw and denoised FPCG signal is reported in Figure 2 that displays raw FPCG signals with three different level of SNR $(-4.4,-16.6$, and $-22.6 \mathrm{~dB}$, grey line) and corresponding denoised FPCG signals after WT filtering (black line), respectively.

Moreover, PCG-Delineator reliably detected location and morphology of the fHSs. Quantitative results relative to the performance of fHSs detection are reported in Table 1. SE and PPV values are both $100 \%$ for S1, and $91 \%$ and $80 \%$, respectively for S2. Eventually, for S1, SE and SNR are associated by a low but significant $(\rho=0.66$, $\mathrm{P}<0.05$ ) correlation. Figure 2 qualitatively showed S1 and S2 sound detection (red and blue bullets, respectively), after the noise level was drastically reduced.

\section{Discussion}

The non-invasive routine measurement of fHSs is of great clinical relevance to assess fetal wellbeing, especially when associated to cardiotocography [18]. Its automatic analysis allows to overcome issues related to
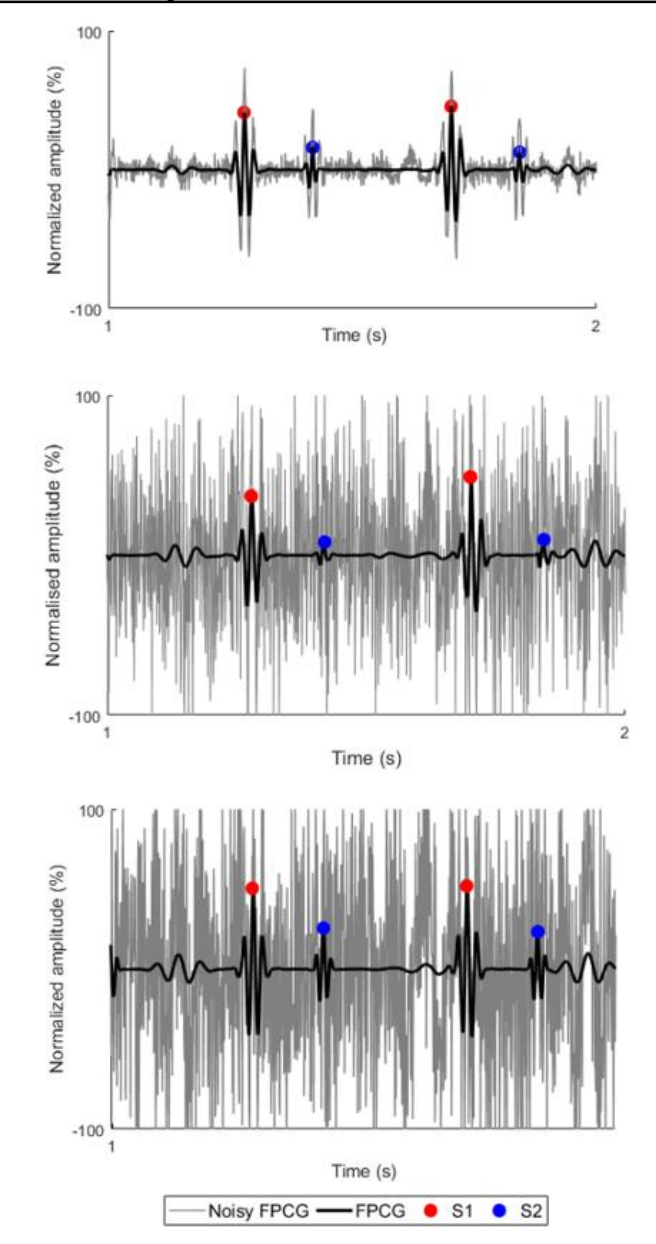

Figure 2. Noisy FPCG signals (in grey) with different SNR: $-4.4 \mathrm{~dB}$ (upper panel), $-16.6 \mathrm{~dB}$ (central panel), and $-22.6 \mathrm{~dB}$ (lower panel). Filtered FPCG signal (in black) with annotations of S1 and S2 (red dot and blue dot, respectively).

Table 1. Statistics of fHSs detection by PCG-Delineator. SE, PPV and $\rho$ (for SEvsSNR and PPVvsSNR) values for $\mathrm{S} 1$ and $\mathrm{S} 2$ are reported.

\begin{tabular}{|c|c|c|}
\hline & S1 & S2 \\
\hline SE $(\%)$ & 100 & 91 \\
& {$[99 ; 100]$} & {$[88 ; 99]$} \\
\hline PPV $(\%)$ & 100 & 80 \\
& {$[100 ; 100]$} & {$[65 ; 85]$} \\
\hline SNR (dB) & 15.9 & 15.9 \\
\hline SEvsSNR & 0.18 & {$[15.2 ; 16.3]$} \\
\hline PPVvsSNR & 0.23 & $0.66^{*}$ \\
\hline
\end{tabular}

*, $\mathrm{P}<0.05$ : statistical significance on the correlation 
subjectivity and physician experience, which may bias diagnoses.

This paper presented a newly developed PCGDelineator as an efficient and advanced algorithm for automatic fHSs from FPCG affected by varying levels of noise. PCG-Delineator has been applied to "Simulated Fetal PCGs database" [6,11] of PhysioNet [12] containing 37 simulated FPCG affected by different levels of noise (original SNR ranged from $-26.7 \mathrm{~dB}$ and $-4.4 \mathrm{~dB}$ ). Original and recomputed SNR values were perfectly correlated $\left(\rho=1.0 ; \mathrm{P}<10^{-200}\right)$, indicating that they provide the same amount of information. To denoise FPCG signals, PCG-Delineator was based on WT approach, using Coiflets mother wavelet (4th order, 7 levels of decomposition). WT filtering significantly increased SNR values $\left(\mathrm{P}<10^{-14}\right)$ and SNR values before and after WT filtering were associated by a low correlation coefficient, indicating that WT filtering is very robust to noise. Denoised FPCG were perfectly aligned (same S1 and S2 location); thus, WT filtering introduces no signal delay and maintains unaltered FPCG characteristics. As shown in Figure 2, noise level was drastically reduced and fHSs becomes always visible; still, some noise survived to filtration, especially in signals initially characterized by very low SNR. In spite of this, PCG-Delineator application provided to accurately detect both $\mathrm{S} 1$ and $\mathrm{S} 2$ from WT-filtered FPCG.

Despite these promising results obtained in this work, future studies are needed with the aim to test PCGDelineator on real FPCG in order to confirm its clinical utility.

\section{Conclusion}

PCG-Delineator is a novel and efficient algorithm for automatic heart sounds detection in FPCG. PCGDelineator efficiently extracts fHS signals from noisy recordings and identifies $\mathrm{S} 1$ and $\mathrm{S} 2$ sounds. Thus, this technique can be a promising fHSs segmentation tool, clearly contributing to better evaluation of the fetal heart functionality.

\section{References}

[1] E. Vollenhoven, and J. Chin, "Phonocardiography: past, present and future," Acta Cardiol., vol. 48, no. 4, pp. 337344, Feb. 1993

[2] F. Kovács, C. Horváth, A. T. Balogh, and G. Hosszú, "Fetal phonocardiography-Past and future possibilities," Comput. Methods Programs Biomed., vol. 104, no. 1, pp. 19-25, Oct. 2011.

[3] V. S. Chourasia, and A. K. Tiwari, "A review and comparative analysis of recent advancements in fetal monitoring techniques," Crit. Rev. Biomed. Eng., vol. 36, no. 5, pp. 335-373, Jan. 2008.

[4] P. Várady, L. Wildt, Z. Benyó, and A. Hein, "An advanced method in fetal phonocardiography," Comput. Methods
Programs Biomed., vol. 71, no. 3, pp. 283-296, July 2003.

[5] V. S. Chourasia, and A. Mittra, "Wavelet-based denoising of fetal phonocardiographic signals," Int. J. Med. Eng. Inform., vol. 2, no. 2, pp. 139, Feb. 2010.

[6] S. Vaisman, S. Y. Salem, G. Holcberg, and A. B. Geva, "Passive fetal monitoring by adaptive wavelet denoising method," Comput. Biol. Med., vol. 42, no. 2, pp. 171-179, Feb. 2012.

[7] S. Sun, Z. Jiang, H. Wang, and Y. Fang, "Automatic moment segmentation and peak detection analysis of heart sound pattern via short-time modified Hilbert transform," Comput Methods Programs Biomed., vol. 114, no. 3, pp. 219-223, May 2014.

[8] S. Schmidt, C. Holst-Hansen, C. Graff, E. Toft, and J. J. Struijk, "Segmentation of heart sound recordings by a duration-dependent hidden Markov model," Physiol. Meas., vol. 31, no. 4, pp. 513-529, Apr. 2010.

[9] D. Springer, L. Tarassenko, and G. Clifford, "Logistic regression-HSMM-based heart sound segmentation," IEEE Trans. Biomed. Eng., vol. 63, no. 4, pp. 822-832, Sep. 2015.

[10] S. D. Min, and H. Shin, "A localization method for first and second heart sounds based on energy detection and interval regulation," J. Electr. Eng. Technol., vol. 10, no. 5, pp. 2126-2134, Sep. 2015.

[11] S. Choi, and J. Zhongwei, "Comparison of envelope extraction algorithms for cardiac sound signal segmentation," Expert Syst. Appl., vol. 34, no. 2, pp. 10561069, Feb. 2008.

[12] A. Misal, and G. R. Sinha, "Denoising of PCG signal by using wavelet transforms," Adv. Comput. Res., vol. 4, no. 1, pp. 46-49, Mar. 2012.

[13] A. Sbrollini et al., "Fetal phonocardiogram denoising by wavelet transformation: robustness to noise," Comput. Cardiol., vol. 44, pp. 1-4, 2017.

[14] M. Cesarelli, M. Ruffo, M. Romano, and P. Bifulco, "Simulation of foetal phonocardiographic recordings for testing of FHR extraction algorithms," Comput. Methods Programs Biomed., vol. 107, no. 3, pp. 513-523, Sep. 2012.

[15] A. L. Goldberger et al., "PhysioBank, PhysioToolkit, and PhysioNet: components of a new research resource for complex physiologic signals," Circulation, vol. 101, no. 23, pp. e215-e220, Jun. 2000.

[16] S. G. Mallat, "A theory for multiresolution signal decomposition: The wavelet representation," IEEE Trans. Pattern Anal. Mach. Intell., vol. 11, no. 7, pp. 674-693, Jul. 1989.

[17] E. Koutsiana, L. J. Hadjileontiadis, I. Chouvarda, and A. H. Khandoker, "Fetal heart sounds detection using wavelet transform and fractal dimension," Front. Bioeng. Biotechnol., vol. 5, Sep. 2017, Art. no. 49.

[18] A. Sbrollini et al., "CTG Analyzer: a graphical user interface for cardiotocography," Conf. Proc. IEEE Eng. Med. Biol. Soc. 2017, pp. 2606-2609, 2017.

Address for correspondence:

Laura Burattini, PhD

Department of Information Engineering,

Università Politecnica delle Marche,

via Brecce Bianche 12, 60131, Ancona, Italy.

E-mail address. 1.burattini@univpm.it 